\title{
Labyrinthe
}

36 | 2011 (1)

«Par les Grecs »

\section{Juifs et Phéniciens}

\section{Martin Bernal}

Traducteur : Laurent Ferri

\section{OpenEdition \\ Journals}

Édition électronique

URL : http://journals.openedition.org/labyrinthe/4130

DOI : $10.4000 /$ labyrinthe.4130

ISSN : 1950-6031

Éditeur

Hermann

Édition imprimée

Date de publication : 15 février 2011

Pagination : 91-102

ISBN : 9782705680541

Référence électronique

Martin Bernal, « Juifs et Phéniciens », Labyrinthe [En ligne], 36 | 2011 (1), mis en ligne le 01 février 2013, consulté le 10 décembre 2020. URL : http://journals.openedition.org/labyrinthe/4130 ; DOI : https:// doi.org/10.4000/labyrinthe.4130

Propriété intellectuelle 


\section{Juifs et Phéniciens}

Martin BERNAL

Professeur émérite de sciences politiques à Cornell University, Martin Bernal est l'auteur de la célèbre trilogie Black Athena, qui a été partiellement traduite en français ${ }^{1}$, et qui avance la thèse d'une origine à la fois égyptienne et phénicienne du "miracle grec». Avant de consacrer plusieurs décennies à sa recherche d'élucidation des racines helléniques, Bernal travaillait principalement sur l'histoire de la Chine à l'époque moderne ${ }^{2}$. Le texte que nous publions, un extrait des mémoires que l'auteur est en train de rédiger, revient sur ce moment de réorientation intellectuelle. L'auteur y présente un premier essai inédit, datant de 1975, qui portait sur les rapports entre Juifs et Phéniciens, et préfigurait en partie l'argumentation de Shlomo Sand ou d'Israël Finkelstein. La réinvention du savant sinisant en antiquisant indiscipliné apparaît ici comme un détour dû à l'abandon, et la requalification, d'une enquête sur l'archéologie de l'identité juive. Bernal situe son entreprise par le biais d'une réflexion autobiographique, où le voyage entre des terrains historiques distincts correspond au périple personnel et professionnel d'un scholar se rendant de Cambridge à Ithaca.

L.D.

\section{Un essai sur les Juifs et les Phéniciens}

À l'automne 1975, je jetai les bases d'un essai dont la première moitié portait sur les ressemblances linguistiques et religieuses entre les cultures « juive » et cananéenne, au nombre desquelles : l'importance du chiffre sept, que l'on peut faire remonter aux Sumériens; le décompte

1. Martin Bernal, Black Athena. The Afroasiatic Roots of Classical Civilization, New Brunswick, Rutgers University Press, 1987-2006, 3 vol. Traduction française des deux premiers volumes sous le titre Black Athena. Les Racines afro-asiatiques de la civilisation classique, Paris, Presses universitaires de France, 1996-1999.

2. Martin Bernal, Chinese Socialism to 1907, Ithaca, Cornell University Press, 1976. 
de la semaine; la circoncision des mâles, empruntée aux Égyptiens; et l'interdiction de manger du porc. Le reste de l'essai concernait la disparition des Phéniciens et l'apparition concomitante des Juifs. J'étais frappé par le fait qu'aucun texte avant Alexandre ne mentionne les Juifs. Au premier siècle de l'ère chrétienne, l'homme politique et écrivain Flavius Josèphe, qui tentait de prouver l'antiquité du peuple juif, avait dépoussiéré de vénérables textes grecs, sans résultat. Dans le même temps, on observe que les Juifs sont très nombreux dans les ouvrages écrits après les conquêtes d'Alexandre. La ligne de démarcation passe entre Aristote, qui ne fait aucune référence aux Juifs, et son disciple Théophraste, qui au contraire mentionne ce peuple assez souvent. On ne saurait attribuer ce changement au fait qu'au cinquième siècle, les écrivains grecs ne voyageaient pas dans des contrées habitées par des Juifs : à preuve Hérodote et Xénophon, pour ne citer que les deux plus connus. Il semblerait en fait qu'il y ait deux raisons, imbriquées, à cette omission. Premièrement, les premiers voyageurs grecs étaient incapables de distinguer les Juifs des autres « Syriens », maintes fois évoqués. La deuxième raison est qu'il y avait moins de Juifs avant Alexandre.

Le judaïsme prit naissance au sein de la communauté d'exilés du royaume de Judée qui s'étaient refugiés à Babylone au début du $\mathrm{vI}^{\mathrm{e}}$ siècle avant l'ère chrétienne. Ils parvinrent à maintenir leur identité culturelle au moyen de l'écriture et de la codification juridique. On pourrait ici faire un parallèle avec les Irlandais du XVII ${ }^{e}$ siècle, qui s'efforcèrent de préserver leur culture dans la production écrite, après leur défaite militaire et politique contre les Anglais; ou avec les Iroquois de la tribu Seneca qui, au XVIII ${ }^{\mathrm{e}}$ siècle et sous l'impulsion de leur chef Handsome Lake ${ }^{3}$, adoptèrent une nouvelle religion et un nouvel alphabet au moment où les Blancs victorieux leur enlevaient leurs terres. Au sixième siècle avant l'ère chrétienne, après soixante ans d'exil, l'empire perse nouvellement triomphant autorisa les Juifs qui le désiraient à retourner à Jérusalem pour y reconstruire leur temple. Ainsi les Juifs disposaient-ils déjà à cette époque de lois religieuses et linguistiques établies pendant l'exil, ainsi que d'une base territoriale.

L'idée conventionnelle selon laquelle tous les Juifs de la Méditerranée descendent génétiquement de cette communauté de base, établie sur un

3. Handsome Lake ou Ganioda'yo (1735-1815), chef de la tribu Iroquoise (ou Haudenosaunee) des Seneca. [Note du traducteur.] 


\section{Juifs et Phéniciens}

territoire d'environ trente kilomètres carrés autour de Jérusalem, ne résiste pas à l'examen. Au premier siècle de notre ère, Philon d'Alexandrie écrivait qu'un million de Juifs vivaient rien qu'en Égypte, sur une population totale estimée par lui à sept millions et demi. Même en admettant que ces chiffres sont exagérés, une telle croissance démographique ne saurait s'expliquer seulement par une filiation génétique.

On sait peu de chose concernant les relations entre les Juifs et Alexandre, deux cents ans avant le retour à Jérusalem. Ce que nous savons, c'est qu'Alexandre détruisit la grande cite phénicienne de Tyr, et que lui et ses successeurs - les princes hellénistiques - brisèrent l'influence phénicienne sur la partie orientale du bassin méditerranéen, qu'elle fût politique ou culturelle (hellénisation). Dans le même temps, les auteurs grecs et juifs commencent à faire référence à des communautés juives dans les mêmes régions : à Chypre, à Cyrène (qui se trouve aujourd'hui en Lybie), à Tarsus (Anatolie), ou à Alexandrie. Toutes ces communautés juives sont apparues dans des zones de peuplement phénicien, à l'exception naturellement d'Alexandrie, fondée par Alexandre sans aucun substrat phénicien. En revanche, Hérodote note la présence d'une importante communauté de Phéniciens de Tyr dans la région de Memphis, capitale traditionnelle de la Basse-Égypte: c'est « le camp des Tyriens. »

Certes, les populations qui se convertissent au judaïsme durant cette période sont nombreuses et variées. Toutefois, ceux qui étaient les plus susceptibles de se convertir étaient les Phéniciens, car ils avaient en commun avec les Juifs de nombreuses coutumes culturelles et religieuses: le langage, la pratique de la circoncision, l'interdiction de manger du porc, etc. Dans le contexte d'une domination culturelle des Macédoniens et des Grecs, il n'est pas surprenant que de nombreux Phéniciens et de nombreux Juifs se soient hellénisés. La menace était réelle, et bien comprise de ceux qui résistaient contre l'acculturation; ainsi Néhémie ${ }^{4}$, qui condamnait les Juifs qui se mariaient à des non-Juifs ou laissaient leurs enfants parler « la langue d'Ashdod », c'est-à-dire le grec. ${ }^{5}$

4. Selon la Bible, Néhémie dirigea la reconstruction des murailles de Jérusalem et son repeuplement par les Juifs. Il est donc considéré par certains comme celui auquel les Juifs doivent la reconstitution de leur entité nationale. [NdT.]

5. Mon assimilation de l'Ashodien au Grec n'est pas communément acceptée. La plupart des auteurs y voient plutôt un dialecte cananéen proche de l'hébreu. Mais mon argument est le suivant: quel autre langage que le grec aurait pu constituer une menace pour l'hébreu ou l'araméen sous domination perse au cinquième siècle? 
$\mathrm{Au}$ lendemain des conquêtes d'Alexandre à la fin du IV siècle, les Phéniciens ne l'aidèrent pas à étendre sa domination à Carthage, qui était leur « colonie ». Cela explique que la langue et la culture phéniciennes s'y soient maintenues pendant des siècles, en dépit des pressions culturelles exercées par les Gréco-Romains en Méditerranée occidentale. La plus puissante des cités phéniciennes dans la région était Carthage, qui subit plusieurs guerres, d'abord contre les Grecs, puis contre les Romains. En dépit de ses extraordinaires talents commerciaux et du brio de ses généraux (Hannibal en particulier), Carthage vit son pouvoir s'éroder de plus en plus, jusqu'à sa défaite finale et sa destruction en -146. D'autres cités phéniciennes ou puniques en Afrique du Nord et en Espagne réussirent à conserver leur liberté à l'intérieur de l'empire romain pendant deux siècles supplémentaires. Or voici ce qu'écrit Strabon de l'Afrique du Nord et de l'Espagne aux alentours du premier siècle de notre ère: «[...] en Europe, les Phéniciens se trouvent posséder aujourd'hui encore les meilleures terres, soit du continent, soit des îles qui en dépendent $»^{6}$ Encore au cinquième siècle, Saint Augustin rapporte que les gens dans son diocèse d'Hippone, aujourd'hui en Algérie, se considèrent comme des Canani, et parlent le Cananéen.

On ne trouve donc aucune trace de Juifs en Méditerranée orientale avant la destruction de Carthage, en - 146. Mais après cette date ils sont au contraire mentionnés en abondance. La comédie de Plaute intitulée Poenulus ou le Petit Carthaginois porte témoignage de la présence à Rome d'une communauté carthaginoise au début du second siècle avant l'ère chrétienne. On y trouve même des dialogues en Cananéen - c'est-à-dire en Phénicien/Hébreu, langue que les spectateurs devaient être capables, sinon de comprendre, du moins d'identifier comme telle. L'adoption par les Romains, en place de leur semaine de neuf jours, d'un système divisant le temps en cycles de sept jours correspondant aux sept « planètes »-jours du Soleil, de la Lune, de Mars, de Mercure, de Jupiter, de Vénus, et de Saturne - est un signe que l'influence sémitique précéda les contacts entre Rome et le judaïsme (ce dernier, au nom du monothéisme, imposa la séquence strictement numérique qui va d'un septième jour vers le suivant). L'existence d'une population juive à Rome est attestée au deuxième siècle avant notre ère par le fait que les

6. Strabon, XVII, 3, 15, trad. A. Tardieu, Hachette, 1880. 


\section{Juifs et Phéniciens}

autorités tentèrent de les en expulser. Ces tentatives échouèrent, et vers la fin dudit siècle, on comptait au moins deux synagogues dans l'Urbs.

D'autres centres urbains phéniciens se convertirent également au judaïsme : ainsi, sur l'île fertile de Djerba, au large de la Tunisie, ou encore à Malte. Le conservatisme culturel et la résistance à la conversion de ces Phéniciens de l'Ouest étaient tels que les premiers Chrétiens disputèrent aux Juifs ces terres de mission. Il est indicatif que Saint Paul, lorsqu'il fit naufrage à Malte, naviguait en direction de l'Espagne, et non en direction de Rome.

La révolte menée par les frères Maccabées en Palestine contre la politique d'hellénisation forcée pratiquée par les Séleucides syro-iraniens $\mathrm{au} \mathrm{II}{ }^{\mathrm{e}}$ siècle avant l'ère chrétienne était la manifestation d'un fondamentalisme religieux. Les deux premiers Livres des Macchabées contiennent le récit des batailles héroïques livrées sur place, mais il est clair que les insurgés durent une grande partie de leur succès à la riche diaspora juive ainsi qu'à Rome, qui étendait alors son influence sur la partie orientale de la Méditerranée. En effet, dans un premier temps, les Romains luttèrent contre les royaumes hellénistiques: dans ce combat, leurs alliés naturels étaient les Juifs, de même que les colonies de population juives en Palestine aidèrent l'Occident à maintenir les nationalismes arabes sous contrôle au $\mathrm{Xx}^{\mathrm{e}}$ siècle. Les Maccabées et la diaspora juive affaiblirent militairement les autorités syriennes, et cette dernière apporta son soutien financier aux Romains, surtout à Jérusalem. Le pèlerinage à Jérusalem faisait alors partie des obligations religieuses pour les Juifs, et le Temple devint une banque internationale extrêmement puissante. Les spécialistes de la persécution des Juifs à cette époque furent surpris par la relative tolérance des Romains vis-à-vis d'une religion qui rejetait à la fois le polythéisme et le culte impérial : c'est ne rien comprendre au basculement géopolitique qui s'était produit au premier siècle après l'ère chrétienne.

Avant le premier siècle, les Romains avaient mis à la tête de Jérusalem des hommes de paille: les Hérodes, issus de la tribu des Iduméens, au sud de la Judée. Bien que leur propre judaïsme fût superficiel, ces rois servirent généralement les intérêts juifs, qu'il s'agît de la Palestine ou de la diaspora. Malheureusement pour eux, après avoir détruit les monarchies hellénistiques, les Romains se détournèrent des Juifs et firent plus que jamais le choix de la culture grecque. Ce fut vrai en particulier de Caligula, même si son successeur Claude fit un retour à la politique antérieure. C'est seulement avec Néron que les Romains s'affirmèrent 
définitivement pro-grecs, anti-juifs, et anti-chrétiens. C'est d'ailleurs sous son règne qu'éclata la première grande Révolte des Juifs. Elle dura six ans, et s'acheva catastrophiquement en 73 avec la destruction du Temple et du système bancaire qui lui était associé - même s'il n'y eut pas d'expulsion massive ou d'exil forcé de la population de Judée.

La diaspora, quant à elle, survit à l'échec de la révolte de 115-117. L'Empereur Trajan fit face dans les provinces nouvellement conquises sur les Parthes à une insurrection de la diaspora juive qui, dans une certaine mesure, était plus grave que la révolte en Palestine. Il y eut plus de victimes de part et d'autre, et, surtout, la destruction complète des Juifs hellénisés fut extrêmement lourde de conséquences. Les historiens n'ont pas prêté à ce phénomène l'attention qu'il mérite, et ce pour deux raisons. D'une part, même avant l'essor du sionisme, les historiens juifs se sont plus préoccupé des événements survenus en Terre Sainte qu'au sein de la diaspora, qu'ils jugeaient moralement inférieure: son sort était par conséquent moins déterminant pour l'histoire des Juifs. D'autre part, la seconde révolte n'eut pas de Flavius Josèphe pour en écrire la brillante chronique.

La troisième grande révolte des Juifs, celle de Bar Kokhba (132136), n'eut pas l'ampleur des deux précédentes, mais elle fut également réprimée avec une grande férocité. Cette fois, interdiction fut faite aux Juifs de vivre à Jérusalem, et la province connue jusqu'alors sous le nom de « Judée » devint « la Palestine ».

Les Chrétiens aussi eurent à souffrir de l'échec de la révolte de la diaspora en 115, mais les conséquences ne furent pas aussi catastrophiques pour eux que pour les Juifs. De fait, il est possible que le Christianisme ait pu servir de refuge précaire à des Juifs forcés de se terrer où ils pouvaient. Le point important est que le centre de gravité du Judaïsme s'était déplacé de la Méditerranée orientale, où ne subsistait plus qu'une petite communauté dans le nord de la Palestine, soit vers l'Est et la Mésopotamie, où les Juifs vivaient sous la protection des Parthes, puis de la dynastie perse des Sassaniens - soit vers l'Ouest, en Espagne et au Maghreb, où les formes occidentales du judaïsme comme du christianisme se ressentirent fortement de l'héritage phénicien.

La suite de mon essai portait sur l'un des éléments de cette culture phénicienne, le sacrifice du premier enfant. À la différence des Égyptiens et de leurs héritiers sur ce point - les Grecs -, les Cananéens faisaient peu 


\section{Juifs et Phéniciens}

de cas de l'immortalité des individus. Leur souci était plutôt d'assurer une descendance nombreuse. De manière symptomatique, Dieu promet à Abraham, non pas l'immortalité, mais de rendre sa postérité nombreuse « comme les étoiles du ciel et comme le sable au bord de la mer ». Le plus grand renoncement qui puisse par conséquent être exigé de lui, c'est le sacrifice de son fils unique et héritier. De nombreuses sociétés pratiquent l'infanticide, soit en tuant, soit en abandonnant sur la place publique les enfants prématurés ou non désirés. Dans la tradition cananéenne, c'est l'inverse: elle requiert le sacrifice de l'enfant le plus précieux, celui qui va assurer la continuité du lignage. Cet enfant, que l'on l'appelle yehid, « le seul et unique », en grec monogenos, « le seul né », est d'autant plus précieux que la mère est trop jeune (Marie) ou trop âgée (Sarah, la mère d'Isaac) pour enfanter. De fait, dans les deux cas, il n'y aura pas d'autre progéniture. Si Dieu promet à Abraham de nombreux descendants, c'est après que celui-ci a offert son fils en sacrifice, et que la substitution avec le bouc a eu lieu. Ce rite n'est pas isolé: ailleurs qu'en Judée, des archéologues ont trouvé côte à côte les restes d'un mouton et ceux d'un d'enfant. En Algérie, une stèle mentionne la substitution mouton/enfant. Toutefois, nous avons les preuves archéologiques que le meurtre rituel d'enfants n'était pas qu'une légende, que ce fût en Palestine, ou dans certaines colonies phéniciennes de Tunisie, de Sicile, ou de Sardaigne.

Le sacrifice était appelé $m l k$. Ce mot rappelant molech ou melek, « le roi », il est donc possible que cette pratique ait été réservée à l'origine aux seuls monarques. Occasion leur était ainsi donnée de manifester leur dévotion à Dieu en tant qu'il symbolisait la cohésion de la communauté. Cependant, des textes latins et grecs, ainsi que des vestiges archéologiques indiquent une « démocratisation » de cette pratique. On attendait de notables, et même de simples citoyens, qu'ils fissent de leurs enfants un sacrifice, afin de sauver la cité lorsqu'elle était menacée d'épidémie, de famine, ou d'attaque par un dangereux ennemi.

Dans la tradition juive, la ligature d'Isaac (Akedah) transcende l'horreur du sacrifice, puisque l'enfant est une offrande refusée. Ce refus est censé traduire la différence essentielle entre les Juifs et les Cananéens païens, mais la démarcation n'est pas absolue; comme je l'ai mentionné plus haut, la substitution d'un mouton est une pratique observée ailleurs dans le monde cananéen. Il en va de même pour le « baptême par le feu » pratiqué à Ge'Hinnom, Gehenna, dans la vallée de Hinnom au sud de Jérusalem. 
Le roi Josué abolit ce rite, qu'il jugeait abominable, au septième siècle - mais ses successeurs revinrent sur nombre de ses réformes.

Le pouvoir de fascination du rite, lui, restait intact, et même après son abolition de fait, il resta un symbole central pour les Juifs. Encore aujourd'hui, chaque premier jour de l'année, les Juifs lisent et chantent le passage de la Bible relatif au sacrifice d'Isaac, perpétré sur le Mont Moriah qui est identifié au site du Temple et donc au centre religieux de l'univers. Du reste, la ligature d'Isaac est aussi un épisode central dans les deux autres religions monothéistes. Pour les chrétiens, l'Akedah est donc une préfiguration du futur sacrifice de Jésus; Abraham était prêt à sacrifier son fils Isaac pour Dieu; Dieu a de même sacrifié Jésus, son fils unique (monogenos) pour sauver le monde. Dans le Coran, il est simplement dit qu'Abraham immola son fils. Les hadiths, recueils de paroles et d'actions attribuées au Prophète, indiquent que le sacrifié n'était pas Isaac mais Ismaël, l'ancêtre des Arabes; selon certains exégètes, le lieu du sacrifice était La Mecque, et non Jérusalem. Le Hajj commémore également l'acte d'Abraham, et les musulmans immolent un mouton voire un chameau pour rappeler son sacrifice.

Chrétiens, Musulmans et Juifs ressentent la même horreur vis-à-vis d'un rite en vigueur chez les Cananéens, alors même que ce rite est symboliquement central dans leurs propres religions. Du coup, la contribution des Cananéens à la civilisation « occidentale », qu'il s'agisse de l'alphabet, ou de la semaine, est généralement sous-évaluée, voire ignorée. L'arriération sinistre des Cananéens et des Phéniciens peut ainsi faire place à la culture lumineuse des Juifs et des Grecs.

\section{La réception de mon essai}

À l'origine, j'avais inclus une version augmentée de la dernière partie dans le premier volume de Black Athena, mais en 1985 je l'en retirai, ne voulant pas provoquer une controverse supplémentaire en marge de ma thèse d'ensemble. Plus tôt, en 1975, alors que je revenais de Cornell University à Cambridge à la fin de l'automne, j'avais fourni un exemplaire de mon texte dans son intégralité à quatre éminents chercheurs avec qui j'avais eu 


\section{Juifs et Phéniciens}

quelques échanges intellectuels préalables : il s'agissait d'Edmund Leach', de Jack Goody ${ }^{8}$, de Moses Finley ${ }^{9}$ et de Meyer Fortes ${ }^{10}$. Comme Edmund était absent lorsque je passai dans son "lodge » de Provost de King's College, quelqu'un me suggéra de laisser mon texte sur son bureau. Le jour suivant je reçus une note incendiaire: comment osais-je le déranger pour ça? Est-ce que je croyais qu'il n'avait rien d'autre à faire que de lire mes arguties? J'accusai d'autant plus le coup qu'Edmund était lui-même connu pour ses audaces intellectuelles, en particulier dans le commentaire des textes bibliques. Deux jours plus tard, il m'envoya une deuxième note, m'invitant cette fois à venir discuter avec lui: mon article lui semblait à présent fort intéressant. Nous eûmes plusieurs heures d'entretiens très fructueux, et Edmund accepta d'écrire une lettre de recommandation pour un poste d'un an comme fellow à la Society for the Humanities de Cornell. Il accepta de me montrer la lettre extraordinairement flatteuse qu'il avait écrite à mon sujet au directeur. Il me comparait à James Frazer ${ }^{11}$, pour qui Trinity College avait créé une fellowship à vie, ce qui lui permit de terminer son énorme et magistral ouvrage Le Rameau d'Or. Même si, aux yeux de Leach, il était improbable, voire impossible, que Cornell pût jamais montrer une pareille générosité, Edmund estimait que j'aurais mérité un traitement identique, et qu'à défaut, une année de bourse serait mieux que rien...

Je bénéficiai également des encouragements et des conseils de Jack Goody - dont le soutien ne s'est pas démenti jusqu'à ce jour. La réaction

7. Sir Edmund Leach (1910-1989), professeur d'anthropologie sociale à Cambridge University (1953-79), nommé Provost de King's College en 1965. De cet auteur, sont notamment disponibles en français: Critique de l'anthropologie, Paris, Presses universitaires de France, 1968; Lévi-Strauss, Paris, Seghers, 1970; Les Systèmes politiques des hautes terres de Birmanie. Analyse des structures sociales kachin, Paris, Maspero, 1972. [NdT.]

8. Sir John [Jack] Goody (né en 1919), professeur d'anthropologie sociale à Cambridge (1954-1984). De cet auteur, sont notamment disponibles en français: La Raison graphique. La domestication de la pensée sauvage, Paris, Minuit, 1978; L'Orient en Occident, Paris, Seuil, 1999; Famille et mariage en Eurasie, Paris, Presses universitaires de France, 2000 ; L'Islam en Europe. Histoire, échanges, conflits, Paris, La Découverte, 2004. [NdT.]

9. Sir Moses Finley (1912-1981), professeur d'études anciennes (1955-1982) à Cambridge. De cet auteur, sont notamment disponibles en français: L'Économie antique, Paris, Minuit, 1975; Le Monde d'Ulysse, Paris, Maspero, 1978; Esclavage antique et idéologie moderne, Paris, Minuit, 1981. [NdT.] 10. Meyer Fortes (1906-1983), professeur d'anthropologie africaine à Cambridge (1950-1973). De cet auteur est disponible en français Edipe et Job dans les religions ouest-africaines, [Tours,] Mame, 1974. [NdT.]

11. James George Frazer (1854-1941) est considéré comme le père fondateur de l'anthropologie religieuse et de la mythologie comparée. Les 12 volumes de son Rameau d'or décrivent des milliers de faits sociaux et religieux, soit relevés par l'auteur sur le terrain ou dans ses lectures, soit relatés par ses correspondants (diplomates, administrateurs coloniaux, explorateurs, missionnaires). [NdT] 
que je redoutais le plus était en fait celle de Moses Finley. Moses s'était refugié à Cambridge au moment du maccarthysme, et il était devenu la figure de proue des « progressistes » dans le milieu des études anciennes. La vieille garde conservatrice, flairant l'intrus, avait accueilli avec méfiance cet Américain, juif de surcroît, et « sachant à peine le grec ». Les jeunes antiquisants, eux, trouvaient formidable que Moses introduise une dose de sciences sociales dans leur discipline. Je fus ravi quand il me dit tout le bien qu'il pensait de mon article - il appréciait surtout ce qu'il voyait comme ma déconstruction de l'identité juive. Il me promit d'écrire lui aussi une lettre de recommandation pour Cornell, lettre que je n'ai pas vue. J'imagine qu'elle était positive pour l'essentiel, en dépit de certaines réserves: d'après Moses en effet, « je ne prouvais rien ». Il me mit aussi en garde contre les risques encourus par les outsiders comme lui et moi: " tu peux écrire tout ce que tu veux, tant que tu ne touches pas à la langue. »

Je retournai le problème. Tout d'abord, je réalisai que je n'étais pas en mesure de « prouver » quoi que ce fût... mais que je n'en avais pas besoin. La recherche de la preuve définitive, au sens mathématique, est vaine en dehors du système euclidien - où elle ne rend d'ailleurs pas que des services. ${ }^{12}$ Dans tous les cas, j'allais à l'encontre d'opinions communément admises mais certainement pas " prouvées». Par conséquent, mon travail ne saurait être jugé uniquement à cette aune. Ensuite, l'avertissement de Finley confirma mon intuition que l'élément langagier était le socle de l'édifice des études anciennes, et qu'il fallait donc s'y intéresser de très près.

La dernière personne à qui j'avais soumis mon essai, Meyer Fortes, était malade à l'époque, et m'écrivit qu'il ne pouvait lui consacrer l'attention qu'il méritait. Toutefois, une de ses remarques ultérieures fit sur moi une profonde impression: il s'étonnait qu'une grande civilisation comme la civilisation égyptienne eût pu avoir si peu d'influence en dehors d'Égypte. Cette remarque me conduisit à examiner si tel était ou n'était pas le cas. À tout prendre, j'étais vraiment ravi de la manière dont mon essai avait été reçu en Angleterre. J'étais plus inquiet concernant la réception qui m'attendait aux États-Unis, en particulier à Cornell.

12. Cf., sur les réserves émises en Inde à l'égard du concept de preuve, C. K. Raju, Cultural Foundations of Mathematics. The Nature of Mathematical Proof and the Transmission of Calculus from India to Europe in the $16^{\text {th }}$ c. CE, New Dehli, Pearson Longman, 2007, p. 3-53. 


\section{Juifs et Phéniciens}

Juste avant de partir pour l'Angleterre au mois de décembre 1974, j'étais allé voir David Owen, le Chair du département de Near Eastern Studies. Comme il était absent, j'avais laissé un exemplaire de mon essai au secrétariat, pour qu'il le lise. Pas de réaction. J'en avais déduit qu'il détestait ma prose, ce qui n'était pas illogique, ce pour au moins deux raisons. Premièrement, j'étais l'exemple même de ce qu'il ne faut pas faire dans l'université: formé et payé pour étudier la Chine moderne, je venais braconner sur les terres des Orientalistes. ${ }^{13}$ Deuxièmement, je contestais la notion de "pureté juive », qui devait être quelque chose d'important pour lui. De crainte d'avoir froissé Owen, j'évitais de le croiser sur le campus. Un jour pourtant il parvint à me coincer, et me dit qu'il aurait plaisir à discuter de mon essai: il me l'aurait d'ailleurs proposé plus tôt s'il avait su mon nom, que j'avais oublié d'indiquer... un lapsus intéressant! Nous eûmes donc une discussion, et il me dit que, puisque j'étais intéressé par les Cananéens, je devrais lire les publications de son maître Cyrus Gordon, et d'un collègue à lui, Michael Astour, qui avaient tous deux travaillé sur les relations entre Grecs et Cananéens.

Au même moment, il se trouva que Susan, future épouse de mon collègue Martin Shefter, prêtait main-forte à un professeur d'histoire à la retraite, Edward Fox, pour la somme qu'il rédigeait. Susan et Martin suggérèrent que Fox, dont je trouvais les idées sur l'interraction entre l'histoire et la géographie fascinantes et stimulantes, pourrait lui aussi être intéressé par mon projet de recherche. Je lui montrai donc mon essai, ainsi qu'à son assistant Leonard Hochberg, et ils furent tellement séduits que Fox écrivit également une lettre en ma faveur au directeur de la Society for the Humanities, qui était alors l'historien des sciences Henry Guerlac. Au nom d'une amitié vieille de plusieurs décennies, il le suppliait de m'accorder une bourse d'études, malgré mon profil pour le moins hétérodoxe. J'ai dans l'idée que cette lettre fit pencher la balance

13. L'éclectisme savant, la curiosité transfrontalière, ainsi qu'un certain non-conformisme « radical», semblent faire partie de l'histoire familiale de Martin Bernal. Son grand-père maternel, Sir Alan Henderson Gardiner (1879-1963), fut l'un des grands égyptologues du xxe siècle, mais ne daigna pas avoir de carrière universitaire. Il ne comprit ni n'approuva le choix de son petit-fils d'étudier le chinois, à la fois à Cambridge et à l'Université de Pékin, où Martin fut l'un des très rares doctorants originaires du monde non communiste, dans les années 1960 - le père John Desmond Bernal (19011971), physicien génial et intellectuel controversé, avait visité Moscou en 1934 et reçu le Prix Staline de la Paix en 1953. Concernant la chronologie intellectuelle de Martin Bernal dans les années 1970, si le changement de cap s'effectua en 1975, c'est bien l'année suivante qu'il publia la version remaniée de sa thèse (soutenue en 1966), Chinese Socialism to 1907. [NdT.] 


\section{Labyrinthe, $n^{\circ} 36$}

en ma faveur. Quoi qu'il en soit, j'obtins la bourse pour l'année suivante, avec comme intitulé de projet: «Le Rôle des Phéniciens dans l'histoire méditerranéenne. » Avoir une année pleine pour faire de la recherche, avec le titre de fellow et les conditions de travail afférentes, me donna la possibilité d'explorer plus avant les pistes que j'avais ouvertes, mais aussi de déplacer mes centres d'intérêt, du côté des " racines grecques » (et plus seulement juives) de la culture européenne.

Traduit de l'anglais par Laurent Ferri 